\title{
Free entry equilibria with positive profits: A unified approach to quantity and price competition games
}

\author{
Rodolphe Dos Santos Ferreira* and Frédéric Dufourt ${ }^{\dagger}$
}

\begin{abstract}
Free entry equilibria are usually characterized by the zero profit condition. We plead instead for a strict application of the Nash equilibrium concept to a symmetric simultaneous game played by actual and potential entrants, producing under decreasing average cost. Equilibrium is then typically indeterminate, with a number of active firms varying between an upper bound imposed by profitability and a lower bound required by sustainability. We use a canonical model with strategies represented by prices, although covering standard regimes of quantity and price competition, to show that in equilibrium the critical (profit maximizing) price must lie between the break-even and the limit prices.
\end{abstract}

Key words Free entry equilibrium, zero-profit condition, oligopolistic competition

JEL classification D43, L13

Accepted 25 February 2007

\section{Introduction}

Free entry is commonly associated with zero profits. Under free entry and exit, positive profits are supposed to stimulate creation of new firms and negative profits to induce destruction of existent firms. A free entry equilibrium might, therefore, be seen as a stationary state, characterized by the zero profit condition, of a dynamic process of net business formation. This view is implicit in the concept of long-run perfectly competitive equilibrium, and is naturally extensive to monopolistic competition, where the relevant scales of individual firms also appear as negligible with respect to market size. As long as profits remain positive, any entrant is then able to reproduce in an unreactive environment the operating conditions and the proceeds of a high number of successful incumbents.

This line of argument ceases to hold, however, when a potential entrant has to compete with a few incumbents only, all producing under internal economies of scale. In this context, a simple replication of the incumbents' performance cannot guarantee identical

\footnotetext{
*BETA-Theme, University Louis Pasteur, Strasbourg, France and Institut Universitaire de France. Email: rdsf@cournot.u-strasbg.fr

${ }^{\dagger}$ BETA-Theme, University Louis Pasteur, Strasbourg, France.

This paper was previously circulated in a larger version with the title "Free entry equilibria: General approach and macroeconomic applications". We thank an anonymous referee for very useful suggestions, as well as Stefano Bosi and Bertrand Wigniolle for their discussions of our paper. We gratefully acknowledge financial support by the Agence Nationale de la Recherche.
} 
success to the entrant, whose environment might be seriously perturbed by that replication. Entry must now be examined as a strategic decision, generally in a complex context, where timing and information considerations are at stake. Faced with this difficulty, a common attitude is to take the zero profit condition as an acceptable approximation, in particular in macroeconomic - or, more widely, general equilibrium - modeling, and to leave more sophisticated approaches, resorting to sequential games with incomplete information, to industrial organization theory. This was already the position adopted in one of the first macroeconomic papers emphasizing the role of increasing returns and imperfect competition, and treating the number of producers as endogenous: "The story [behind the solution concept based on the zero profit condition] can only be defended as an approximation. Entry and exit are complicated phenomena, involving difficult game theoretic issues that defy neat analytic formulation" (Weitzman 1982, p. 797). So, we seem to be trapped in a dilemma: either to force a solution concept devised for non-strategic forms of competition into the domain of oligopoly, or to resort to industrial organization tools that might prove too complex and also too specific for an accurate macroeconomic or general equilibrium use. The point we want to make in the present paper is that we are by no means doomed to that dilemma. A straightforward application of the concept of Nash equilibrium to static symmetric games reproducing standard regimes of oligopolistic competition offers in fact a simple way out.

We owe to Shubik $(1959,1984)$ the idea that entry can be modeled as a simultaneous game between actual and potential entrants, depicted as "firms-in-being". At an equilibrium of such a game, along with active profit maximizing firms, there might be inactive firms that optimally decide not to produce, on the basis of correct conjectures about the actions of the former. This asymmetry might be the consequence of some advantage of incumbents over potential entrants, creating a barrier to entry. However, it can also prevail in a completely symmetric game where all players are a priori indistinguishable. As a matter of fact, ex ante symmetry is required for an accurate representation of a perfectly contestable market, characterized by costless entry and exit and no disadvantage for potential entrants relative to incumbents (Baumol et al. 1982).

For a market to be perfectly contestable any observable profile of incumbents' strategies must be sustainable; that is, no potential entrant may be able to make a profit by becoming active. This requirement might suggest that, as in the dynamic story of business formation, equilibrium profits are necessarily close to zero as soon as entry is free, or the market perfectly contestable. This is indeed true if incumbents' capacity to earn positive profits extends to any potential entrant, always in a position to attract enough customers, either by simply imitating incumbents' behavior when firms are small relative to the market, or by slightly undercutting incumbents' prices when competition takes place in the undifferentiated Bertrand oligopoly. An example of the former alternative is competition within the Chamberlinian "large group", where an individual price decision has no sensible repercussions on the industry price level, as in Dixit and Stiglitz (1977). Another example is Cournot competition in an undifferentiated industry, provided the optimal scale of each firm is small with respect to market size, so that the Cournot equilibrium outcome approximates the long-run perfectly competitive outcome, characterized by efficiency and zero profits, as in Novshek (1980). Yano (2006) offers an example of the latter alternative. He uses the same 
type of free entry equilibrium concept as Novshek (i.e. a Nash equilibrium of a single stage game played in a perfectly contestable market), but, by assuming Bertrand-like competition in an undifferentiated oligopoly, ${ }^{1}$ he obtains the long-run perfectly competitive outcome without assuming a small firm to market ratio. At the same time, he provides a solid game theoretic foundation to the theory of contestable markets.

These examples suggest that sustainability is not independent of the regime of competition. Instead of the "price sustainability" appearing in the original definition of Baumol et al. (1982), which implicitly refers to Bertrand competition, we may think of a "quantity sustainability" referring to Cournot competition, with different implications (Brock and Scheinkman 1983; Brock 1983). However, preferably, rather than simply opposing price and quantity sustainability, we may completely divorce the concept of sustainability from the specific regime of competition to which it applies (d'Aspremont et al. 2000). Indeed, along with Cournot competition, price competition within a "small group" producing differentiated goods is another situation where the zero profit condition is not implied by sustainability, and where multiple free entry equilibria may exist in addition to the one at break-even prices. In such equilibria, the strategies of active firms entail positive profits and are nevertheless sustainable because potential entrants, taking those strategies as given, realize that, whatever they do, demand will be insufficient for attaining the scale at which production becomes profitable. Also, under these circumstances, there is no sensible reason for the incumbents to accommodate entry.

The objective of the present paper is to provide a unified conceptual and analytical framework for the study of free entry equilibria, covering different regimes of oligopolistic competition and different specifications of internal increasing returns. Our starting point is the same as in d'Aspremont et al. (2000): we take as the relevant concept of free entry equilibrium the standard Nash solution of a simultaneous symmetric non-cooperative game, such that active and inactive firms coexist. Sustainability is then just the optimizing condition that applies to inactive firms, given their correct conjectures about the decisions of active firms. However, in the present paper, we go a step further in the way of unification: we build a canonical model, where firms strategies are always represented by prices, but which covers different regimes of both price and quantity competition. One advantage of this comprehensive representation is that free entry equilibria can then be generally characterized by the interval between the break-even and the limit prices, to which the critical price maximizing the incumbent profit should belong. This interval induces a range of numbers of active firms that are compatible with a free entry equilibrium: a type of indeterminacy appearing as a robust property of oligopolistic competition in contestable markets.

Our paper is organized as follows. We present our conceptual framework in Section 2, by: (i) defining the concept of free entry equilibrium; (ii) introducing the canonical model where strategies are represented by prices; and (iii) establishing equilibrium conditions on

\footnotetext{
${ }^{1}$ As a matter of fact, in the game designed by Yano (2006), firms simultaneously choose a price and a set of quantities that are equivalent from the point of view of the profits they generate. Together with free entry, this device allows him to extend Bertrand equilibrium to the case where the average cost function is not constant, without incurring the inexistence problem pointed out by Edgeworth. Yano (2005) extends this analysis to encompass strategic technological choices.
} 
incumbents' prices under general specifications of cost and demand functions. These conditions are then applied in Section 3 to standard regimes of quantity and price competition in homogeneous and differentiated markets, respectively, and the corresponding outcomes are compared. We conclude in Section 4.

\section{Oligopolistic competition with free entry}

The analysis carried out in this section comprises three steps. In Subsection 2.1, we introduce a fairly general, although simple, game theoretic framework applying to perfectly contestable oligopolistic markets, and exploiting symmetry of strategy profiles (in the spirit of Cooper and John 1988). In this context, we use in a standard way the equilibrium solution of a simultaneous and symmetric game to define the concept of free entry equilibrium, under the additional requirement that some firms optimally decide to remain inactive. Two conditions characterize equilibrium: profitability and sustainability. In Subsection 2.2, we propose a canonical model covering different regimes of competition in which incumbents' strategies are always represented by prices, even when quantities, or locations in some characteristics space, are involved (as in Cournotian or spatial competition, respectively). We further formulate general assumptions on the cost and demand functions. In Subsection 2.3, we translate the two conditions characterizing a free entry equilibrium into the requirement that the common price chosen by the incumbents be a critical point of their profit function above the break-even price (for profitability) and below the limit price (for sustainability). These bounds on incumbents' prices translate in turn into a non-degenerate admissible interval to which the number of active firms should belong.

\subsection{Concept of free entry equilibrium}

Free entry means absence of any entry barrier accounting for some advantage of incumbents over potential entrants. Under free entry all firms, whether established or not, are supposed to benefit from full equality of opportunities. However, this does not imply that they are assured of equality of results. In game theoretic terms, firms are assumed to play a symmetric game (equality of opportunities), the equilibria of which need, however, not be symmetric (possible inequality of results). Equilibria might display a primary kind of asymmetry (the one which concerns us here) involving the distinction between active and inactive firms. A free entry equilibrium is just a Nash equilibrium of the symmetric game, such that some firms are active and some inactive. In other words, there is at least a potential entrant optimally deciding not to actually enter .

This free entry equilibrium is usually viewed as a sub-game perfect equilibrium of a two-stage game, with firms deciding at the first stage either to enter or not, and then with entrants competing at the second stage according to some specified regime (typically, quantity or price competition). The first stage entry decision, possibly implying the same sunk cost for any entrant, does, however, not give the right to an equal treatment at the second stage, as implicitly assumed. Under internal increasing returns, there may be active and inactive entrants at a second stage equilibrium. Consequently, we might as well resort 
to a simultaneous game, and take entry/exit decisions as implicit in quantity and/or price decisions (Novshek 1980; d'Aspremont et al. 2000; Corchón and Fradera 2002; Yano 2005, 2006).

To be explicit, consider a symmetric simultaneous game played by $N$ competing oligopolistic firms, each one with the same strategy space $\mathbb{S}$ and the same payoff function $\Pi: \mathbb{S}^{N} \rightarrow \mathbb{R}$. A firm is inactive if it chooses an element of the subset $\mathbb{S}_{0}$ of strategies that lead to zero output, and it is active if it chooses a strategy in the complementary subset. The nature of the subset $\mathbb{S}_{0}$ results from the particular specification of the model, $\mathbb{S}_{0}$ being for instance equal to $\{0\}$ in quantity competition games, or to the set of prices higher than any customer's reservation price in price competition games. We admit that the payoff function is constant with respect to any of its arguments over $\mathbb{S}_{0}$, if this set has more than one element. Now consider strategy profiles $\mathbf{s} \in \mathbb{S}^{N}$ that are symmetric within the class of $n$ active firms $(0<n<N),{ }^{2}$ all choosing $s_{n} \in \mathbb{S} \backslash \mathbb{S}_{0}$ while $N-n$ inactive firms indifferently choose some element of $\mathbb{S}_{0}$. It is clear that the relevant information in $\mathbf{s}$ is completely contained in the pair $\left(s_{n}, n\right)$. Similarly, as the vector $\mathbf{s}_{-i} \in \mathbb{S}^{N-1}$ of strategies of the $N-1$ competitors of any firm $i$ has $n-\delta$ elements equal to $s_{n}$ (with $\delta=1$ if firm $i$ is active and $\delta=0$ if it is inactive) and $N-1-(n-\delta)$ elements belonging to $\mathbb{S}_{0}$, it can be fully characterized by the triplet $\left(s_{n}, n, \delta\right)$. The profit $\boldsymbol{\Pi}\left(s_{i}, \mathbf{s}_{-i}\right)$ of any firm $i$, choosing strategy $s_{i}=s$ and facing a profile $\mathbf{s}_{-i}$ of its competitors' strategies with such characterization, can then be denoted accordingly by $\Pi\left(s, s_{n}, n, \delta\right)$.

If we apply the Nash equilibrium concept to this framework, for a pair $\left(s_{n}, n\right)$ to characterize an equilibrium, the profit $\Pi\left(s, s_{n}, n, 1\right)$ of an active firm must reach a maximum at $s=s_{n}$, and the profit $\Pi\left(s, s_{n}, n, 0\right)$ of an inactive firm must reach a maximum at any $s_{0} \in \mathbb{S}_{0}$. Furthermore, if we take free entry as comprehending free exit, so that sunk costs are excluded, ${ }^{3}$ inactivity always results in zero profits, so that any equilibrium $\left(s_{n}, n\right)$ must verify $\Pi\left(s_{n}, s_{n}, n, 1\right) \geq 0$ and $\Pi\left(s_{0}, s_{n}, n, 0\right)=0$.

In the usual approach, ${ }^{4}$ if $n$ is the number of firms having chosen to enter at the first stage, the two conditions for a free entry equilibrium $\left(s_{n}, n\right)$ without sunk costs are: first, that $\Pi\left(s, s_{n}, n, 1\right)$ reach a maximum non-negative value on $\mathbb{S} \backslash \mathbb{S}_{0}$ at $s=s_{n}$, and second, that whenever this value is positive there be no equilibrium with $n+1$ entrants (symmetric with respect to all of them). The second condition means that, for any strategy $s_{n+1} \in \mathbb{S} \backslash \mathbb{S}_{0}$, if $\Pi\left(s, s_{n+1}, n+1,1\right)$ is maximized on $\mathbb{S} \backslash \mathbb{S}_{0}$ at $s=s_{n+1}$ then $\Pi\left(s_{n+1}, s_{n+1}, n+1\right.$, $1)<0$. Putting together these two conditions one obtains the zero profit condition commonly seen as implied by free entry $\left(\Pi\left(s_{n}, s_{n}, n, 1\right)=\max _{s \in \mathbb{S} \backslash \mathbb{S}_{0}} \Pi\left(s, s_{n}, n, 1\right)=0\right)$, provided one neglects the so-called "non-integer problem" (as $n$ belongs to $\mathbb{N}^{*}$, not to $\mathbb{R}_{+}, \Pi\left(s_{n+1}, s_{n+1}, n+1,1\right)<0<\Pi\left(s_{n}, s_{n}, n, 1\right)$ is in fact the generic case).

Instead, we use the standard definition of a Nash equilibrium of a single stage game, just characterizing separately, as best responses, the decisions of active and inactive firms:

\footnotetext{
${ }^{2}$ We admit that $n>0$ in order to eliminate trivial equilibria, and that $n<N$ to put aside the case where entry is impossible for lack of further participants.

${ }^{3}$ Exclusion of sunk costs is consistent with our assumption of a simultaneous game, but is not crucial. In a two-stage game, if all (actual) entrants incur the same positive sunk cost $k$ at a first stage, the profit of an inactive entrant $-k$ is negative, instead of 0 , but this translation does not fundamentally alter the argument.

${ }^{4}$ See Götz (2005) for a recent example.
} 
Definition 1 A non-trivial symmetric free entry equilibrium is a pair $\left(s_{n}, n\right)$ in $\left(\mathbb{S}_{\mathbb{1}} \backslash \mathbb{S}_{0}\right) \times$ $\{1, \ldots, N-1\}$ satisfying two conditions:

$$
\begin{aligned}
& \max _{s \in \mathbb{S}} \Pi\left(s, s_{n}, n, 1\right)=\Pi\left(s_{n}, s_{n}, n, 1\right) \geq 0 \text { (profitability) and } \\
& \max _{s \in \mathbb{S}} \Pi\left(s, s_{n}, n, 0\right)=\max _{s \in \mathbb{S}_{0}} \Pi\left(s, s_{n}, n, 0\right)=0 \text { (sustainability). }
\end{aligned}
$$

For a strategy profile characterized by the pair $\left(s_{n}, n\right)$ to be an equilibrium, it must be profitable for any active firm to choose the strategy $s_{n}$, meaning that no higher profit is attainable either while staying active $\left(\Pi\left(\cdot, s_{n}, n, 1\right)\right.$ is maximized at $\left.s_{n}\right)$ or through becoming inactive ( $\Pi\left(s_{n}, s_{n}, n, 1\right)$ is non-negative). The strategy profile must also be sustainable with respect to inactive firms, meaning that these firms should not be able to obtain a positive profit by becoming active.

\subsection{A canonical pricing model}

To be more specific and still cover different regimes of competition with a unified framework, we now introduce a simple model where strategies, whatever their nature, can be represented by prices. Take an industry producing either a homogeneous or a composite good sold at price $P$. Demand for this good is given by a twice differentiable decreasing function $D:(0, \widetilde{P}) \rightarrow(0, \infty)$, with $\widetilde{P} \in(0, \infty]$, continuously extended for $P \geq \widetilde{P}$ as $D(P)=0$. The good is potentially produced by $N$ firms, under internal increasing returns, with the same twice differentiable increasing cost function $C:(0, \infty) \rightarrow(0, \infty)$, extended to $C(0)=0$ (no sunk costs) and such that average cost $C(y) / y$ is decreasing on $(0, \infty)$. As in the preceding subsection, we restrict our analysis to equilibria that are symmetric with respect to $n$ active firms $(0<n<N)$, all choosing the same strategy $s_{n} \in \mathbb{S} \backslash \mathbb{S}_{0}$. This strategy will always be represented by the price $p_{n}$ at which any active firm intends to sell its output. Therefore, any firm deciding to supply quantity $y$ at price $p$, and facing demand $d\left(p, p_{n}, n, \delta\right)$, has to solve a problem that can be stated as follows:

$$
\max _{(p, y) \in \mathbb{R}_{+}^{2}}\left\{p y-C(y): y \leq d\left(p, p_{n}, n, \delta\right)\right\} .
$$

Clearly, a pair $(p, y)$ such that $0<y<d\left(p, p_{n}, n, \delta\right)$ cannot be a solution to this problem, because the profit is increasing in $y$ if $C(y) / y \leq p$. Therefore, the firm will always decide either to produce $y=d\left(p, p_{n}, n, \delta\right)$ or to stay inactive (i.e. to choose $\left.y=0\right)$, so that we can directly refer to the canonical program (CP) in the single decision variable $p$

$$
\max _{p \in \mathbb{R}_{+}}\left\{p d\left(p, p_{n}, n, \delta\right)-C\left(d\left(p, p_{n}, n, \delta\right)\right)\right\}
$$

and then check that the maximum profit is non-negative, taking otherwise $y=0$ as the optimal decision.

One sees immediately that the CP covers the case where firms produce differentiated goods and compete in prices. It is less evident yet true that it also covers for instance the case of a homogeneous oligopoly with Cournotian firms. Indeed, given symmetry with respect 
to $n$ active firms, each one of these firms chooses $p_{n}=P$ and $y_{n}=D(P) / n$, whereas $N-n$ inactive firms all choose $y=0$. The residual demand at price $p$ for any firm, whether active $(\delta=1)$ or inactive $(\delta=0)$ is $D(p)-(n-\delta) y_{n}=D(p)-(1-\delta / n) D\left(p_{n}\right) \equiv d\left(p, p_{n}, n, \delta\right)$. As $y=d\left(p, p_{n}, n, \delta\right)$ if and only if $D(p)=(n-\delta) y_{n}+y$, the CP is indeed equivalent to the standard program of the Cournotian firm; namely, $\max _{y \in \mathbb{R}_{+}}\left\{D^{-1}\left((n-\delta) y_{n}+y\right) y-\right.$ $C(y)\}$.

The specific form of contingent demand to the firm $d\left(\cdot, p_{n}, n, \delta\right)$, of which the Cournotian contingent demand is just an example, depends on the assumed regime of competition. However, we can establish a general relation between the demand to the active firm $d\left(p_{n}, p_{n}, n, 1\right)$ as a function of $p_{n}$ (the price set by all the $n$ active firms) and the demand to the industry:

$$
n \alpha(n) d\left(p_{n}, p_{n}, n, 1\right)=D(P), \text { with } P=p_{n} / \alpha(n),
$$

where $\alpha(n)$ is a positive aggregating factor to be used when the product is a composite good $(\alpha(n) \equiv 1$, otherwise $) .^{5}$

We now introduce two general assumptions on the cost and demand functions. The first expresses existence of internal increasing returns to scale (yet not at an increasing rate). Formally: ${ }^{6}$

Assumption 1 The function $C(y) / y$ is twice differentiable and has a negative, non-decreasing elasticity $\left(\epsilon_{y} C(y)-1<0, \epsilon_{y y}^{2} C(y) \geq 0\right)$ in the interval $(0, \infty)$.

The second assumption is introduced to ensure that the profit function is well-behaved and has adequate boundary properties, namely, that it takes negative values when the scale of production is either too small or too large. It further ensures that the demand to any firm, hence its average revenue, is larger for the incumbent than for the potential entrant (as the former competes with only $n-1$ producing firms, whereas the latter faces one more competitor). Formally:

Assumption 2 For any triplet $\left(p_{n}, n, \delta\right)$, the function $d\left(\cdot, p_{n}, n, \delta\right)$ is twice differentiable in the interval $\left(0, \widetilde{p}\left(p_{n}, n, \delta\right)\right)$ in which it is positive (where $\widetilde{p}\left(p_{n}, n, \delta\right) \in(0, \infty]$ is the supremum of customers' reservation prices), and has in this interval a negative, decreasing elasticity $\left(\epsilon_{p} d\left(\cdot, p_{n}, n, \delta\right)<0, \epsilon_{p p}^{2} d\left(\cdot, p_{n}, n, \delta\right)>0\right)$, such that

$$
\begin{aligned}
& \frac{1}{\lim _{p \rightarrow 0} \epsilon_{p} d\left(p, p_{n}, n, \delta\right)}<\lim _{y \rightarrow \infty} \epsilon_{y} C(y)-1 \text { and } \\
& \frac{1}{\lim _{p \rightarrow \widetilde{p}\left(p_{n}, n, \delta\right)} \epsilon_{p} d\left(p, p_{n}, n, \delta\right)}>\lim _{y \rightarrow 0} \epsilon_{y} C(y)-1 .
\end{aligned}
$$

\footnotetext{
${ }^{5}$ Deflating the price $p_{n}$ by the aggregating factor in the definition of the price index, $P$ (the price of the composite good), ensures that the aggregate expenditure, $P D(P)$, in the industry is indeed equal to the sum $n p_{n} d\left(p_{n}, p_{n}, n, 1\right)$ of firms revenues. A well-known example of the use of such price and quantity indices for industries producing a composite commodity is afforded by the Dixit and Stiglitz (1977) framework (where $\alpha(n)=n^{1 /(\sigma-1)}$, with $\sigma$ the constant elasticity of substitution between elementary goods). For a more general analysis, see d'Aspremont et al. (2007).

${ }^{6}$ We denote $\epsilon_{x} f(x, y) \equiv(\partial f(x, y) / \partial x) x / f(x, y)$ the partial elasticity of $f$ at $(x, y)$ with respect to $x$. All related elasticity notations are self-explanatory.
} 


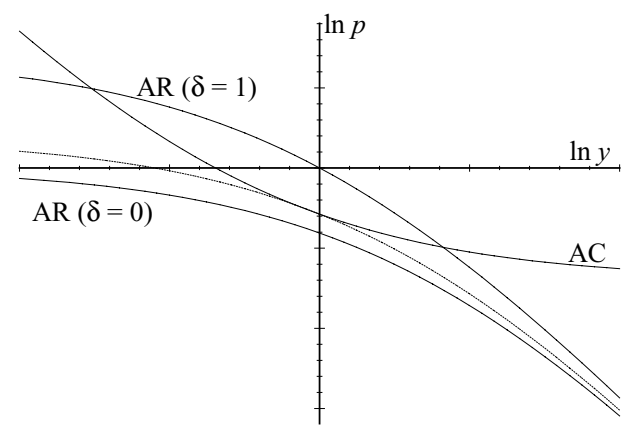

Figure 1 Average cost (AC) and revenue (AR) curves.

Furthermore, for any $p$, the function $d(p, \cdot)$ is increasing in $p_{n}$ and $\delta$, and non-increasing in $n$, as long as its value remains positive.

Geometrically, as illustrated by Figure 1, Assumption 1 states that the average cost curve, $C(y) / y$, is decreasing and convex when represented on a diagram with logarithmic scales (see curve AC, with slope $\epsilon_{y} C(y)-1$ ). Assumption 2 states that the average revenue curve (given by the inverse of the function $d\left(\cdot, p_{n}, n, \delta\right)$ ) is decreasing, strictly concave, and always lower than the average cost curve for an output either close to zero or close to infinity (see curves AR, with slopes $1 / \epsilon_{p} d\left(p, p_{n}, n, \delta\right)$ ).

In this diagram, the profitability condition requires that the average revenue curve of an active firm $\operatorname{AR}(\delta=1)$ be higher than the average cost curve, AC, for intermediate values of $y$, whereas the sustainability condition requires that the average revenue curve of an inactive firm, $\operatorname{AR}(\delta=0)$, be lower than the average cost curve, AC, for all values of $y$. The two conditions are compatible because average revenue is increasing in $\delta$ by Assumption 2 . The curve that is tangent to the average cost curve corresponds to the limit case of a price $p_{n}$ such that a potential entrant can at most make a zero profit if it decides to become active. We will come back to this limit price in the following subsection.

As a last remark, it should be noticed that twice differentiability of the function $d\left(\cdot, p_{n}, n, \delta\right)$ is more than a technical assumption. Indeed, it excludes the case of Bertrand competition (price competition in a homogeneous oligopoly) because $d\left(\cdot, p_{n}, n, \delta\right.$ ) has then a discontinuity at $p=p_{n}$.

\subsection{Equilibrium conditions}

Under the assumptions of the preceding subsection, we can reformulate the profitability and the sustainability conditions in terms of the price $p_{n}$ set (or targeted) by all the $n$ active firms. Profitability requires that this price be an interior solution to the CP (hence, a critical point of the corresponding profit function $\left.\Pi\left(\cdot, p_{n}, n, 1\right)\right)$, and that this critical price be at least equal to the break-even price, entailing zero profits. By contrast, sustainability requires that the critical price be at most equal to the limit price deterring entry. In the following, we 
are going to give formal definitions of these three reference prices, and examine sufficiency of the above conditions.

Definition 2 A critical price $p_{n}^{*}$ is a positive price that, when simultaneously set by $n$ active firms, satisfies the first order condition necessary for an interior solution of CP; that is, solves the equation of marginal revenue with marginal cost:

$$
p_{n}\left(1+1 / \epsilon_{p} d\left(p_{n}, p_{n}, n, 1\right)\right)=C^{\prime}\left(d\left(p_{n}, p_{n}, n, 1\right)\right) .
$$

If the critical price $p_{n}^{*}$ entails non-negative profits, this first order condition is in fact sufficient, under our assumptions, for an interior solution of the $\mathrm{CP}$, in spite of the possible lack of quasi-concavity of $\Pi\left(\cdot, p_{n}, n, 1\right)$, because of the non-convexity of the cost function. Profitability is then satisfied at $p_{n}^{*}$, as stated in the following lemma:

Lemma 1 (Profitability I) Under Assumptions 1 and 2, the symmetric strategy profile represented by the pair $\left(p_{n}^{*}, n\right) \in \mathbb{R}_{++} \times\{1, \ldots, N-1\}$ satisfies the profitability condition if and only if $p_{n}^{*}$ is a critical price entailing non-negative profits or, equivalently, leading to a revenue-cost ratio at least equal to one:

$$
g\left(p_{n}^{*}, n\right) \equiv \frac{p_{n}^{*} d\left(p_{n}^{*}, p_{n}^{*}, n, 1\right)}{C\left(d\left(p_{n}^{*}, p_{n}^{*}, n, 1\right)\right)} \geq 1 .
$$

ProOF: See Appendix.

Now, notice that for an increasing function $g(\cdot, n)$, condition (PNNC) can equivalently be expressed by requiring that the critical price be at least equal to the break-even price $\underline{p}(n)$, which entails zero profits, or equivalently leads to a unit value of the revenue-cost ratio. We formalize and develop this idea in the following. To begin with, we give a general definition of the break-even price, independently of $g(\cdot, n)$ being an increasing function. To understand this definition, recall that $\widetilde{P}$ is the price, possibly infinite, at which demand becomes nil, and that the price $P$ of the composite good is equal to $p_{n}$ deflated by the aggregating factor $\alpha(n)$.

Definition 3 The break-even price $p(n)$, is the lowest price $p_{n}$ that, when set by all the $n$ active firms, allows them to get non-negative profits: $\underline{p}(n) \equiv \inf \mathcal{P}(n)$, with

$$
\mathcal{P}(n) \equiv\left\{p_{n} \in(0, \alpha(n) \widetilde{P}): g\left(p_{n}, n\right) \geq 1\right\}
$$

(by convention, $\underline{p}(n)=\infty$ if $\mathcal{P}(n)=\varnothing$ ).

As just observed, the profitability requirement can be equivalently expressed by the inequality $p_{n}^{*} \geq \underline{p}(n)$ (where $p_{n}^{*}$ is a critical price), provided $g(\cdot, n)$ is an increasing function, a property that is verified when the demand to the industry has an elasticity that is always at least equal to -1 . Otherwise, the market revenue, $P D(P)$, may decrease with $P$, imposing an upper bound on the set $\mathcal{P}(n)$, so that the preceding inequality is only a necessary (but in general not sufficient) condition for profitability. However, under the following additional assumption, implying in particular quasi-concavity of the 
function $g(\cdot, n)$, that inequality remains a sufficient condition for profitability, as stated in Lemma 2.

Assumption 3 Demand to the industry has an elasticity $\epsilon_{P} D(\cdot)$ that is non-increasing whenever smaller than -1 . The elasticity $\epsilon_{y} C^{\prime}(y)$ of marginal cost is larger, for any $y$, than the least upper bound $1 / \lim _{P \rightarrow \widetilde{P}} \epsilon_{P} D(P)$ of the elasticity of inverse demand.

Lemma 2 (Profitability II) Under Assumptions 1, 2 and 3, the symmetric strategy profile represented by the pair $\left(p_{n}^{*}, n\right) \in \mathbb{R}_{++} \times\{1, \ldots, N-1\}$ satisfies the profitability condition if and only if $p_{n}^{*}$ is a critical price at least equal to the break-even price: $p_{n}^{*} \geq \underline{p}(n)$.

ProOF: See Appendix.

Finally, we introduce the concept of limit price, to be taken as un upper bound imposed on the critical price so as to ensure sustainability. This price is defined as "the highest common price which the established seller(s) believe they can charge without inducing at least one increment to entry" (Bain 1949, p. 454). This is the price leading to an average revenue curve of the potential entrant that is just below the average cost curve (Modigliani 1958), as represented by the curve that is tangent to curve AC in Figure 1. Formally:

Definition 4 The limit price, $\bar{p}(n)$, is the highest price $p_{n}$ that, when set by all the $n$ active firms, prevents an inactive firm from getting positive profits: $\bar{p}(n) \equiv \sup \overline{\mathcal{P}}(n)$, with

$$
\overline{\mathcal{P}}(n) \equiv\left\{p_{n} \in(0, \alpha(n) \widetilde{P}): \max _{p \in\left(0, \widetilde{p}\left(p_{n}, n, 0\right)\right)} G\left(p, p_{n}, n\right) \leq 1\right\},
$$

with $G\left(p, p_{n}, n\right) \equiv \frac{p d\left(p, p_{n}, n, 0\right)}{C\left(d\left(p, p_{n}, n, 0\right)\right)}$ and $\widetilde{p}\left(p_{n}, n, 0\right)$ as defined in Assumption 2.

Observe that the elasticity with respect to $p$ of the revenue-cost ratio $G$ is

$$
\epsilon_{p} G\left(p, p_{n}, n\right)=1+\left(1-\epsilon_{y} C\left(d\left(p, p_{n}, n, 0\right)\right)\right) \epsilon_{p} d\left(p, p_{n}, n, 0\right) .
$$

Hence, by inequalities (3) and (4) in Assumption 2, this elasticity is positive for $p$ close to zero and negative for $p$ close to $\widetilde{p}\left(p_{n}, n, 0\right)$, implying that $G\left(\cdot, p_{n}, n\right)$ has indeed an interior maximum. Therefore, we can determine the limit price, $\bar{p}(n)$, as the solution in $p_{n}$ to equations:

$$
\begin{gathered}
p d\left(p, p_{n}, n, 0\right)=C\left(d\left(p, p_{n}, n, 0\right)\right) \\
-\epsilon_{p} d\left(p, p_{n}, n, 0\right)=\frac{1}{1-\epsilon_{y} C\left(d\left(p, p_{n}, n, 0\right)\right)} ;
\end{gathered}
$$

namely, the zero profit condition and the first order condition for maximization of $G\left(\cdot, p_{n}, n\right)$, respectively.

Given Definition 4, we can now reformulate the sustainability condition by reference to the limit price, $\bar{p}(n)$.

Lemma 3 (Sustainability) Under Assumptions 1 and 2, the condition $p_{n} \leq \bar{p}(n)$ is necessary and sufficient for the pair $\left(p_{n}, n\right) \in \mathbb{R}_{++} \times\{1, \ldots, N-1\}$ to satisfy the sustainability condition. 


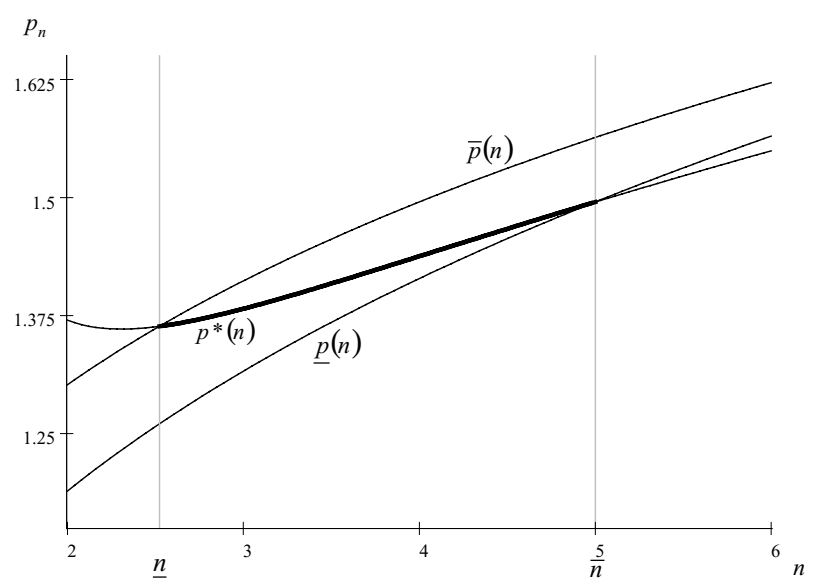

Figure 2 Critical, limit and break-even prices.

Proof: See Appendix.

We summarize in the following proposition the results stated in the two last lemmata.

Proposition 1 Under Assumptions 1, 2 and 3, a symmetric profile represented by the pair $\left(p_{n}^{*}, n\right) \in \mathbb{R}_{++} \times\{1, \ldots, N-1\}$ is a free entry equilibrium if and only if $p_{n}^{*}$ is a critical price between the break-even price and the limit price: $\underline{p}(n) \leq p_{n}^{*} \leq \bar{p}(n)$.

Figure 2 provides a representation of this equilibrium condition in the space $\left(n, p_{n}\right)$, where the critical, break-even and limit prices appear as functions $p^{*}(\cdot), \underline{p}(\cdot)$ and $\bar{p}(\cdot)$ of a real (instead of an integer) number $n .^{7}$ Observe how the condition $p(n) \leq p_{n}^{*} \leq \bar{p}(n)$, resulting in the thick segment of the critical price curve, translates into a restriction on the number $n$ of active firms. This number should belong to the interval $[\underline{n}, \bar{n}]$, with endpoints defined by $p^{*}(\underline{n})=\bar{p}(\underline{n})$ and $p^{*}(\bar{n})=\bar{p}(\bar{n})$.

Obviously, as soon as the interval $[\underline{n}, \bar{n}]$ contains more than one integer, the profitability and sustainability conditions are compatible with existence of free entry equilibria with positive profits, along with the one determined by the zero profit condition (corresponding to a number of active firms equal to the highest integer in the interval, precisely equal to $\bar{n}$ in this particular example). It is worthwhile emphasizing that this source of equilibrium multiplicity differs from the one usually considered in the coordination failures literature and stemming from the seminal paper of Cooper and John (1988). In this literature, multiple symmetric equilibria are associated with the same exogenous number $n=N$ of players (thus resulting, in our framework, in a multi-valued function $\left.p^{*}(\cdot)\right)$. Such multiplicity relies on

\footnotetext{
${ }^{7}$ The case represented in Figure 2 corresponds to price competition in a differentiated oligopoly with a constant elasticity of substitution aggregator (Dixit-Stiglitz), where we have assumed a unit-elastic demand to the industry, with constant expenditure normalized to unity, a constant elasticity of substitution within the industry equal to 6 , and a constant elasticity of the marginal cost function equal to -0.2 . To facilitate comparisons between regimes of competition, we have chosen the parameter values so as to ensure that the critical and break-even price functions intersect at the same integer value $\bar{n}$.
} 
strategic complementarity, resulting in the requirement that the best response of $p$ be an increasing function of $p_{n}$. No such condition is necessary in our approach, because symmetry is now imposed only within each class of active and inactive firms. This explains why, as we are going to show, equilibrium multiplicity can prevail even under strategic substitutability, as it is typically the case under Cournot competition.

\section{Regimes of competition}

To illustrate the potential of our analytical framework, we now apply it to two standard regimes of quantity and price competition. As for quantity competition, we limit our analysis to the Cournot homogeneous oligopoly. As for price competition, we must refer to a market for differentiated products, because the Bertrand homogeneous oligopoly is outside our scope and leads, at free entry equilibrium, to the competitive outcome (Yano 2005, 2006). We might for instance use the well-known Dixit and Stiglitz (1977) model, modified so as to account for manipulability of the industry price index by each one of a "small group" of active firms (d'Aspremont et al. 1996). However, because this model has already been treated in this perspective by d'Aspremont et al. (2000), we shall devote our analysis of price competition to the Salop (1979) spatial model, where the strategy variables include, besides prices, locations in the characteristics space.

\subsection{Quantity competition with product homogeneity: the Cournot model}

There are two sources of decreasing average cost; namely, the presence of a fixed cost and the existence of internal economies of scale accounting for decreasing marginal cost. These two sources are not equivalent, as attested by their specific effects in the DixitStiglitz model, where multiple free entry equilibria generally exist when marginal cost is an isoelastic decreasing function, but never when it is constant, in the presence of a fixed cost (d'Aspremont et al. 2000). ${ }^{8}$ However, in the two regimes of competition we are going to analyze, the weaker source of decreasing average cost is sufficient to ensure multiplicity of free entry equilibria and, hence, to illustrate the use of our framework. For simplicity, we shall accordingly assume a positive fixed (non sunk) cost, $\phi$, and a constant positive marginal cost, normalized to one: $C(y)=\phi+y$ if $y>0$ and $C(0)=0$, a function that clearly satisfies Assumption 1. On the demand side we assume, also in the analysis of both regimes, a unitelastic demand to the industry, $D(P)=b / P$, with $b>0$, satisfying Assumption $3 .^{9}$ One advantage of this specification is that the break-even price becomes independent of the particular regime of competition we are considering, because expenditure $b$ in the industry is not affected by price changes. The break-even price is then given by

$$
\underline{p}(n)=\frac{1}{1-n \phi / b},
$$

\footnotetext{
${ }^{8}$ In the latter case, the admissible interval $[\underline{n}, \bar{n}]$ is still non-degenerate, but it contains at most one integer, the one determined by the zero profit condition.

${ }^{9}$ Equilibrium with a single active firm will consequently be excluded.
} 
an increasing function of the number $n$ of active firms and of the share $\phi / b$ of individual fixed cost in aggregate expenditure.

With this specification of the demand to the industry and according to what has already been shown in Subsection 2.2, the Cournotian contingent demand can be expressed in a symmetric configuration as

$$
d\left(p, p_{n}, n, \delta\right)=b\left(\frac{1}{p}-\frac{1-\delta / n}{p_{n}}\right),
$$

where $p$ is the market price aimed at by the firm and $p_{n}=b / n y_{n}$ the price representing the strategy $y_{n}$ expected from each one of its $n-\delta$ active competitors (with $\delta=1$ if the firm is itself active, $\delta=0$ otherwise). This function has a partial elasticity with respect to $p$ (for $p$ in the interval $\left(0, p_{n} /(1-\delta / n)\right)$ in which individual demand is positive and finite) given by

$$
\epsilon_{p} d\left(p, p_{n}, n, \delta\right)=-\frac{1}{1-(1-\delta / n)\left(p / p_{n}\right)} .
$$

It is easy to check that it satisfies Assumption 2, and that $\epsilon_{p} d\left(p_{n}, p_{n}, n, 1\right)=-n$.

From (11), we see that the contingent demand to the inactive firm is independent of the number $n$ of active firms, so that the limit price is itself constant in $n:{ }^{10}$

$$
\bar{p}(n)=\frac{1}{(1-\sqrt{\phi / b})^{2}},
$$

again an increasing function of the share $\phi / b$ of individual fixed cost in aggregate expenditure. As to the critical price, it is simply equal to the markup factor $\mu(n)=n /(n-1)$ on marginal cost (multiplied by 1 , the normalized marginal cost):

$$
p^{*}(n)=\frac{n}{n-1} \text {. }
$$

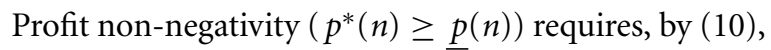

$$
n \leq \sqrt{b / \phi} \equiv \bar{n}
$$

an upper bound that must be at least equal to 2 , for a free entry equilibrium to exist, so that $\phi / b \leq 1 / 4$ is a necessary condition for existence. Sustainability $\left(p^{*}(n) \leq \bar{p}(n)\right)$ in turn requires:

$$
n \geq \frac{\bar{n}}{2-1 / \bar{n}} \equiv \underline{n}
$$

Notice that the admissible interval $[\underline{n}, \bar{n}]$ contains more than one integer for $\bar{n} \geq 3$; that is, for a small enough degree of economies of scale, as determined by the share $\phi / b$ of individual fixed cost in aggregate expenditure, which should not exceed $1 / 9$. The degree of indeterminacy increases with $\bar{n}$; that is, it is larger the smaller the degree of economies

\footnotetext{
${ }^{10} \operatorname{Using}(11)$ with $\delta=0$, we get from $(8) p y=b\left(1-p / p_{n}\right)=\phi+y$, so that $p_{n}=[b /(b-(\phi+y))][(\phi+y) / y]$. From (9), we get $p / p_{n}=y /(\phi+y)$. Hence, $\phi+y=\sqrt{\phi b}$, and, finally, $p_{n}=1 /(1-\sqrt{\phi / b})^{2}$.
} 


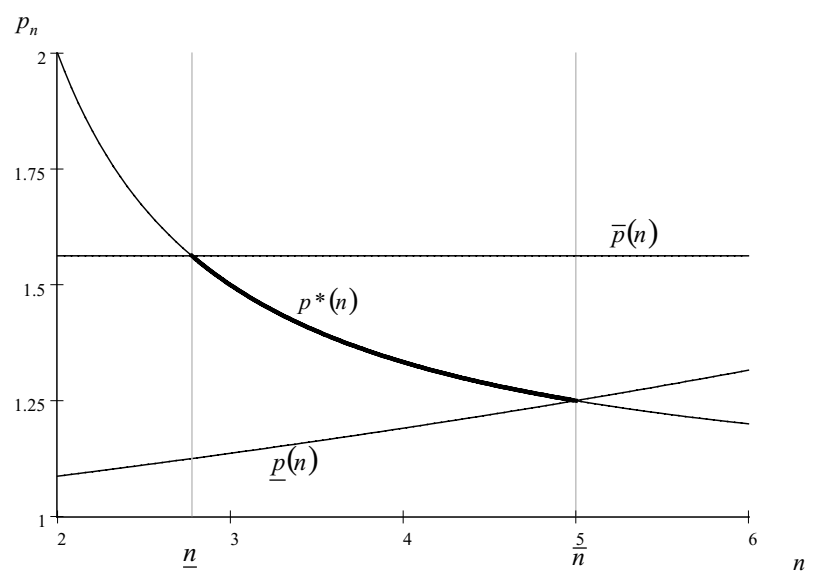

Figure 3 Equilibrium conditions in the Cournot model.

of scale. However, a low degree of economies of scale results in a relatively large number of active firms, reducing the impact of variations in $n$ on the markup factor (and, hence, on the equilibrium price). Figure 3 gives an illustration (for $\phi / b=0.04$ and similarly to Figure 2) of the conditions demanded from the critical, break-even and limit prices to ensure profitability and sustainability. For the sake of a comparison with the price competition regime, notice that the set of candidate numbers of active firms in a free entry equilibrium is $\{3,4,5\}$ in this particular case.

\subsection{Price competition with strategic product differentiation: the Salop model}

In the industrial organization literature, spatial competition is a popular alternative to nonaddress models relying on constant elasticity of substitution or quadratic consumers' utility functions. Although less frequent in macroeconomic modeling, it has, for instance, already been used by Weitzman (1982), who introduced a macroeconomic version of the Salop (1979) model of the circular city. The space of characteristics of the industry good is represented by a circle with perimeter equal to 1 , on which consumers' locations are uniformly distributed with unit density. A consumer devoting a positive budget $b$ to the purchase of that good and located at point $x$ between two firms $j$ and $j+1$, which are themselves located at $a_{j}$ and $a_{j+1}$, respectively, will buy from firm $j$ if $p_{j}+\tau\left(x-a_{j}\right)<p_{j+1}+\tau\left(a_{j+1}-x\right)$, where $p_{j}$ and $p_{j+1}$ are the prices set by the two firms and $\tau$ is the subjective transportation rate in money equivalent units. The marginal consumer who is indifferent between the two suppliers is the one located at point $x_{(j, j+1)}=\left(a_{j}+a_{j+1}\right) / 2+\left(p_{j+1}-p_{j}\right) / 2 \tau$, so that the market area of firm $j$ is

$$
x_{(j, j+1)}-x_{(j-1, j)}=\frac{a_{j+1}-a_{j-1}}{2}+\frac{\left(p_{j-1}+p_{j+1}\right) / 2-p_{j}}{\tau},
$$


which is independent upon its own location $a_{j}$. However, although indifferent about its precise location within its market area, firm $j$ is assumed to set its price, $p_{j}$, on the basis of its conjectures not only about prices $p_{j-1}$ and $p_{j+1}$ but also about the locations $a_{j-1}$ and $a_{j+1}$ simultaneously chosen by its neighbors. This implies in particular that, when inactive at the strategy profile taken as reference, a deviating firm does not conjecture that the locations of the two competitors between which it decides to locate are going to be benevolently accommodated in response to its decision to deviate into activity. As a consequence, any deviating firm is able to manipulate its market area through its pricing decision, within the segment separating its two neighbors, but the length of this segment is $2 / n$ if the firm is active and only $1 / n$ if it is inactive (assuming that locations are symmetric with respect to the $n$ active firms).

On the basis of symmetry with respect to both locations and prices, we obtain from (17) the following expression for contingent demand to the representative firm (with $\delta=1$ if it is active, $\delta=0$ otherwise):

$$
d\left(p, p_{n}, n, \delta\right)=(b / \tau p)\left((1+\delta) \tau / 2 n+p_{n}-p\right),
$$

with $p \in\left(0,(1+\delta) \tau / 2 n+p_{n}\right]$. The partial elasticity of $d\left(p, p_{n}, n, \delta\right)$ with respect to $p$ is:

$$
\epsilon_{p} d\left(p, p_{n}, n, \delta\right)=-\frac{(1+\delta) \tau / 2 n+p_{n}}{(1+\delta) \tau / 2 n+p_{n}-p} .
$$

All the conditions of Assumption 2 are again satisfied.

Because $\epsilon_{p} d\left(p_{n}, p_{n}, n, 1\right)=-\left(1+n p_{n} / \tau\right)$, the markup factor on the marginal cost is $\mu=1+\tau / n p_{n}$, so that we obtain the following expression for the critical price as a (decreasing) function of $n$ :

$$
p^{*}(n)=\mu^{*}=\frac{1}{2}+\sqrt{\frac{1}{4}+\frac{\tau}{n}} .
$$

Profit non-negativity $\left(p^{*}(n) \geq \underline{p}(n)\right)$ imposes, by this equation and equation (10), an upper bound on the number $n$ of active firms:

$$
n \leq \frac{\tau}{\sqrt{\tau \phi / b}(1+\sqrt{\tau \phi / b})} \equiv \bar{n},
$$

which must be at least equal to 2 for existence of a free entry equilibrium, so that the ratio $\phi / b$ cannot be too large. The upper bound $\bar{n}$ on the number of active firms is increasing in the transportation rate, $\tau$ (representing the degree of product differentiation), and decreasing in the share $\phi / b$ of individual fixed cost in aggregate expenditure (determining the degree of internal economies of scale). Finally, equations (8) and (9) lead to the limit price $^{11}$

$$
\bar{p}(n)=(1+\sqrt{\tau \phi / b})^{2}-\tau / 2 n,
$$

\footnotetext{
${ }^{11}$ Using (18) with $\delta=0$, we obtain from (8) $p y=(b / \tau)\left(\tau / 2 n+p_{n}-p\right)=\phi+y$. Also, using (19) with $\delta=0$, we get from (9): $p /\left(p_{n}-p+\tau / 2 n\right)=y / \phi$. These equations together entail: $y=\sqrt{b \phi / \tau}$ and $p=1+\sqrt{\tau \phi / b}$, and then $p_{n}=(1+\sqrt{\tau \phi / b})^{2}-\tau / 2 n$.
} 


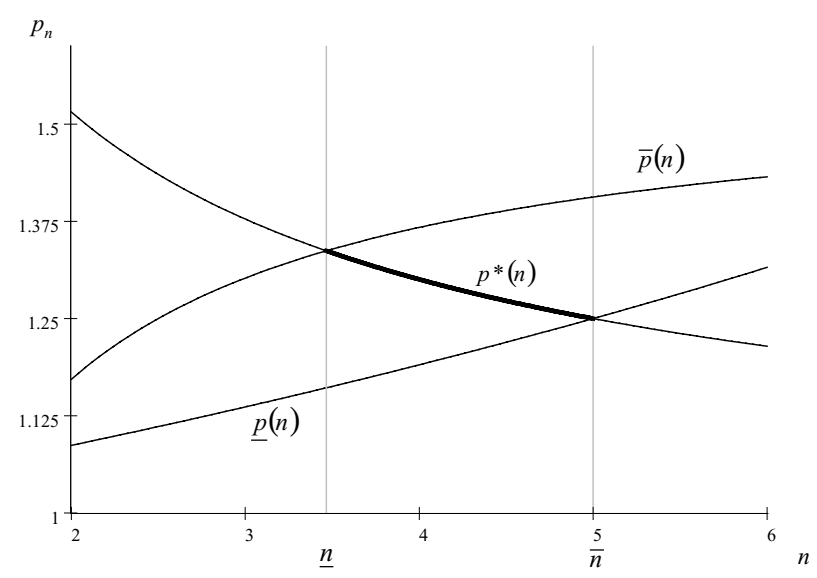

Figure 4 Equilibrium conditions in the Salop model.

and, after a straightforward computation, to the lower bound imposed by sustainability $\left(p^{*}(n) \leq \bar{p}(n)\right)$ :

$$
n \geq \frac{\tau}{\left(\sqrt{2(1+\sqrt{\tau \phi / b})^{2}+1 / 4}-1\right)^{2}-1 / 4} \equiv \underline{n} .
$$

It can easily be checked that the ratio $\underline{n} / \bar{n}$ between the endpoints of the admissible interval $[\underline{n}, \bar{n}]$ is a decreasing function of the variable $\sqrt{\tau \phi / b}$, which tends to $3 / 4$ as this variable tends to zero. As a consequence, the admissible interval contains at least two integers if $\bar{n} \geq 4$, which requires a small enough share of individual fixed cost in aggregate expenditure $(\phi / b<1 / 4)$ and a high enough degree of product differentiation as measured by $\tau$. We represent in Figure 4 (for the same value 0.04 of the ratio $\phi / b$ and for $\tau=25 / 16$, so as to get the same $\bar{n}=5$ and the same $p^{*}(\bar{n})=\mu(\bar{n})=1.25$ as in Figure 3$)^{12}$ the free entry equilibrium conditions in terms of the critical, break-even and limit prices. Observe that the set of numbers of active firms compatible with free entry equilibrium is now $\{4,5\}$, a proper subset of the corresponding set under quantity competition.

We can now formulate a tentative conclusion of the analysis performed in this section. The degree of internal economies of scale (here determined by the share $\phi / b$ of the individual fixed cost in the aggregate expenditure) must be low enough to ensure existence, and even more so to entail multiplicity, of free entry equilibria. However, as the share $\phi / b$ becomes smaller, the amplitude of price variations across equilibria becomes smaller too. Under price competition, existence and especially multiplicity also require a high enough degree of product differentiation (here represented by the rate of transportation $\tau$ ) and a correspondingly high degree of market power. Sustainability (as indicated by the level of the lower bound $\underline{n}$ on the admissible number of active firms, given the same upper bound $\bar{n}$ ) is harder to ensure under price competition than under quantity competition,

\footnotetext{
${ }^{12}$ Imposing either the same upper bound $\bar{n}$ or the same corresponding critical price $p^{*}(\bar{n})=p(\bar{n})$ leads to the relation: $\sqrt{\tau}=1 /(1-\sqrt{\phi / b})$. The choice of the numerical value $\phi / b=0.04$ then implies $\tau=25 / 16$.
} 
where potential entrants expect incumbents to stick to specific output levels. However, price sustainability remains compatible with positive profits under strategic product differentiation, where potential entrants at least expect incumbents to stick to specific locations in the characteristics space. In the non-address model of Dixit and Stiglitz, where potential entrants have no disadvantage relative to incumbents as regards the capacity to benefit from product differentiation, the presence of a fixed cost is, however, not enough to ensure sustainability along with positive profits.

\section{Conclusion}

We have argued in this paper that, in spite of an almost universal convention, zero profits should not be imposed as an equilibrium condition under free entry, beyond the realm of non-strategic forms of competition. A straightforward application of the Nash equilibrium concept to standard simultaneous symmetric games, portraying diverse regimes of oligopolistic competition, typically entails multiple free entry equilibria with various adjacent numbers of active firms and different levels of positive profits. These equilibria are characterized by two conditions: profitability (the price should be no smaller than the break-even price) and sustainability (the price should be no larger than the limit price). These conditions define a non-degenerate interval of admissible numbers of active firms, that typically contains more than one integer. The zero profit condition then appears as no more than a particular selection criterion, picking up the least profitable equilibrium, associated with the highest integer in this interval. Our analytical framework and the indeterminacy results that it allows us to establish are quite robust, and apply to both quantity and price competition; although the latter regime, in particular under non-strategic forms of product differentiation, makes sustainability harder to attain when profits are positive.

Beyond industrial organization, the indeterminacy of free entry equilibrium has potentially significant macroeconomic implications. In particular, it raises a coordination problem and, consequently, favors the emergence, even under dynamic determinacy, of sunspot fluctuations. These might be induced by some extrinsic, potentially varying, public signal on which firms in each industry need to coordinate (Dos Santos Ferreira and Dufourt 2006; Dos Santos Ferreira and Lloyd-Braga 2003). Also, taking into account this particular type of indeterminacy enlarges the scope for coordination failures, which cease in particular to depend upon strategic complementarity.

\section{Appendix}

\section{Proof of Lemma 1 (Profitability I)}

Take a pair $\left(p_{n}^{*}, n\right) \in \mathbb{R}_{++} \times\{1, \ldots, N-1\}$. Equation (FOC) in Definition 2 (i.e. the equality of marginal revenue and marginal cost) is the necessary first order condition for an interior solution of the CP. Hence, the condition that $p_{n}^{*}$ be a critical price entailing non-negative profits is clearly necessary for profitability. Let us examine if it is sufficient. A sufficient second order condition for a local maximum is that the marginal revenue decrease faster with $y$ (increase faster with $p$ ) than the marginal cost; that is, that the elasticity with respect to $p$ of 
the left-hand side of (FOC) be larger than the corresponding elasticity of the right-hand side:

$$
1-\frac{\epsilon_{p p}^{2} d\left(p_{n}^{*}, p_{n}^{*}, n, 1\right)}{1+\epsilon_{p} d\left(p_{n}^{*}, p_{n}^{*}, n, 1\right)}>\epsilon_{y} C^{\prime}\left(d\left(p_{n}^{*}, p_{n}^{*}, n, 1\right)\right) \epsilon_{p} d\left(p_{n}^{*}, p_{n}^{*}, n, 1\right)
$$

By Assumption 2 and given (FOC) (implying $\left.1+\epsilon_{p} d\left(p_{n}^{*}, p_{n}^{*}, n, 1\right)<0\right)$, the left-hand side of inequality (SOC) is larger than one, so that the inequality is satisfied if the right-hand side is not larger than one. This is always the case if marginal cost is non-decreasing. Otherwise, if $\epsilon_{y} C^{\prime}\left(y^{*}\right)<0$ (with $y^{*}=d\left(p_{n}^{*}, p_{n}^{*}, n, 1\right)$ ), the profit non-negativity condition (PNNC), by (FOC) and Assumption 1, can be expressed for a critical price as

$$
\epsilon_{p} d\left(p_{n}^{*}, p_{n}^{*}, n, 1\right) \geq \frac{1}{\epsilon_{y} C\left(d\left(p_{n}^{*}, p_{n}^{*}, n, 1\right)\right)-1}
$$

This inequality implies that the right-hand side of (SOC) is indeed at most equal to

$$
\frac{\epsilon_{y} C^{\prime}\left(y^{*}\right)}{\epsilon_{y} C\left(y^{*}\right)-1}=1+\frac{\epsilon_{y y}^{2} C\left(y^{*}\right)}{\epsilon_{y} C\left(y^{*}\right)-1} \leq 1,
$$

by Assumption 1, thus verifying condition (SOC). Hence, the profit function has a local interior non-negative maximum at any critical price $p_{n}^{*}$ satisfying (PNNC). This maximum is in fact a global maximum. Indeed, by differntiability of the profit function, if there were two maxima, they would be separated by a minimum satisfying (FOC) and violating (SOC), hence (PNNC) (and ( $\left.\mathrm{PNNC}^{\star}\right)$ ). However, if profit has a negative minimum at some price, then it cannot have a positive maximum at a higher price, because $\epsilon_{p} d\left(\cdot, p_{n}^{*}, n, 1\right)$ (in the left-hand side of $\left.\left(\mathrm{PNNC}^{\star}\right)\right)$ is decreasing and $\epsilon_{y} C\left(d\left(\cdot, p_{n}^{*}, n, 1\right)\right)$ (in the right-hand side) is non-increasing, so that $\left(\mathrm{PNNC}^{\star}\right)$ cannot be satisfied at this higher price.

\section{Proof of Lemma 2 (Profitability II)}

Using equation (2) relating demand to the active firm and demand to the industry, we can compute the elasticity with respect to $p_{n}$ of the revenue-cost ratio $g\left(p_{n}, n\right)$ defined in Lemma 1:

$$
\epsilon_{p_{n}} g\left(p_{n}, n\right)=1+\left(1-\epsilon_{y} C\left(D\left(p_{n} / \alpha(n)\right) / n \alpha(n)\right)\right) \epsilon_{P} D\left(p_{n} / \alpha(n)\right) .
$$

There are two cases. If $\epsilon_{P} D(P) \in[-1,0)$ for any $P \in(0, \widetilde{P})$, this elasticity is positive by Assumption 1 . The function $g(\cdot, n)$ is then increasing, so that the condition $g\left(p_{n}, n\right) \geq 1$ in Lemma 1 can be immediately replaced by the condition $p_{n} \geq \underline{p}(n)$, with $g(\underline{p}(n), n)=1$. If $\epsilon_{P} D(P)<-1$ for some $P$, the elasticity $\epsilon_{p_{n}} g\left(p_{n}, n\right)$ might be non-positive. The right-hand side of (24) is then non-increasing in $p_{n}$, either through the elasticity of the cost function (by Assumption 1), or through $\epsilon_{P} D\left(p_{n} / \alpha(n)\right)$ (by Assumption 3). Hence, once non-positive, the elasticity $\epsilon_{p_{n}} g\left(p_{n}, n\right)$ can never become positive again. In other words, the function $g(\cdot, n)$ is quasi-concave, and the set $\mathcal{P}(n)$ of prices entailing non-negative profits, defined by (5), is an interval.

Next, we show that the joint profit $\widehat{\Pi}(P, n) \equiv P D(P)-n C(D(P) / n \alpha(n))$, computed under the constraint that every firm produce the same quantity, is increasing at a price $P=p_{n}^{*} / \alpha(n)$, such that $p_{n}^{*}$ is a critical price. Consider the derivative with respect to $P$ of the joint profit function:

$$
\frac{\partial \widehat{\Pi}(P, n)}{\partial P}=D(P)\left[1+\left(\frac{\alpha(n) P-C^{\prime}(D(P) / n \alpha(n))}{\alpha(n) P}\right) \epsilon_{P} D(P)\right]
$$


and replace $\alpha(n) P$ by $p_{n}^{*}$, using the first order condition that defines a critical price:

$$
\begin{aligned}
\frac{\partial \widehat{\Pi}\left(p_{n}^{*} / \alpha(n), n\right)}{\partial P} & =D\left(p_{n}^{*} / \alpha(n)\right)\left[1-\frac{\epsilon_{P} D\left(p_{n}^{*} / \alpha(n)\right)}{\epsilon_{p} d\left(p_{n}^{*}, p_{n}^{*}, n, 1\right)}\right] \\
& =D\left(p_{n}^{*} / \alpha(n)\right)\left[-\frac{\epsilon_{p_{n}} d\left(p_{n}^{*}, p_{n}^{*}, n, 1\right)}{\epsilon_{p} d\left(p_{n}^{*}, p_{n}^{*}, n, 1\right)}\right] .
\end{aligned}
$$

By Assumption 2, this derivative is positive. Also, $\partial \widehat{\Pi}(P, n) / \partial P$ becomes negative at zero by Assumption 3 , because $\epsilon_{P} D(P)$ is non-increasing and since $\epsilon_{y} C^{\prime}(D(P) / n \alpha(n)) \epsilon_{P} D(P)<1$ for any $P$. Hence, the joint profit function $\widehat{\Pi}(\cdot, n)$ is (strictly) quasi-concave, so that $\sup _{P \in(0, \widetilde{P})} \widehat{\Pi}(P, n)>p_{n}^{*} / \alpha(n)$. Also, $\sup _{P} \widehat{\Pi}(P, n)$, unless infinite, must belong to the set $\mathcal{P}(n)$ of prices entailing non-negative profits. In any case, as this set is an interval, $\underline{p}(n) \leq p_{n}^{*}<\alpha(n) \sup _{P} \widehat{\Pi}(P, n)$ implies: $p_{n}^{*} \in \mathcal{P}(n)$.

\section{Proof of Lemma 3 (Sustainability)}

As $d(p, \cdot, n, 0)$ is increasing (by Assumption 2), and $C(y) / y$ is decreasing in $y$ (by Assumption 1), the revenue-cost ratio $G\left(p, p_{n}, n\right)$ is increasing in $p_{n}$. By definition of the limit price $\bar{p}(n), G(\widehat{p}, \bar{p}(n), n)=1$ at $\widehat{p}$ maximizing $G(\cdot, \bar{p}(n), n)$. Hence, $p_{n} \leq \bar{p}(n)$ is clearly a necessary condition for sustainability $\left(G\left(\widehat{p}, p_{n}, n\right)>1\right.$ if $\left.p_{n}>\bar{p}(n)\right)$. For sufficiency, consider $p_{n}<\bar{p}(n)$ and $G\left(p, p_{n}, n\right)>1$ for some $p$. Then, $G(p, \bar{p}(n), n)>1$, contradicting the definition of $\bar{p}(n)$.

\section{References}

d'Aspremont, C., R. Dos Santos Ferreira, and L.-A. Gérard-Varet (1996), “On the Dixit-Stiglitz model of monopolistic competition," American Economic Review 86, 623-29.

d'Aspremont, C., R. Dos Santos Ferreira, and L.-A. Gérard-Varet (2000), "Contestability and the indeterminacy of free entry equilibria," Japanese Economic Review 51, 72-83.

d'Aspremont, C., R. Dos Santos Ferreira, and L.-A. Gérard-Varet (2007), "Competition for market share and for market size: Oligopolistic competition with varying competitive toughness," International Economic Review 48, 761-84..

Bain, J. S. (1949), “A note on pricing in monopoly and oligopoly,” American Economic Review 39, 448-64.

Baumol, W., J. Panzar, and R. Willig (1982), Contestable Markets and the Theory of Industry Structure, New York: Harcourt Brace Jovanovich.

Brock, W. A. (1983), “Contestable markets and the theory of industry structure: A review article," Journal of Political Economy 91, 1055-66.

Brock, W. A., and J. A. Sheinkman (1983), "Free entry and the sustainability of natural monopoly: Bertrand revisited by Cournot," D. S. Evans, ed, Essays on Industrial Organization and Regulation, 231-52, Amsterdam: North-Holland.

Corchón, L. C., and I. Fradera (2002), “Comparative statics in Cournot free entry equilibrium,” Mathematical Social Sciences 44, 155-68.

Cooper, R., and A. John (1988), “Coordinating coordination failures in Keynesian models,” Quarterly Journal of Economics 103, 441-63.

Dixit, A., and J. Stiglitz (1977), “Monopolistic competition and optimum product diversity,” American Economic Review 67, 297-308.

Dos Santos Ferreira, R., and F. Dufourt (2006), "Free entry and business cycles under the influence of animal spirits," Journal of Monetary Economics 53, 311-28. 
Dos Santos Ferreira, R., and T. Lloyd-Braga (2003), "Business cycles with free entry ruled by animal spirits," Center for Economic Policy Research Discussion Paper no. 3919, Centre for Economic Policy Research, London, UK.

Götz, G. (2005), “Market size, technology choice, and the existence of free-entry Cournot equilibrium,” Journal of Institutional and Theoretical Economics 161, 503-21.

Modigliani, F. (1958), “New developments on the oligopoly front,” Journal of Political Economy 66, $215-32$.

Novshek, W. (1980), "Cournot equilibrium with free entry," Review of Economic Studies 47, 473-86.

Salop, S. (1979), "Monopolistic competition with outside goods," Bell Journal of Economics 10, 141-56.

Shubik, M. (1959), Strategy and Market Structure, New York: John Wiley.

Shubik, M. (1984), A Game-Theoretic Approach to Political Economy, Cambridge, MA: MIT Press.

Weitzman, M. (1982), "Increasing returns and the foundations of unemployment theory," Economic Journal 92, 787-804.

Yano, M. (2005), "Coexistence of large firms and less efficient small firms under price competition with free entry," International Journal of Economic Theory 1, 167-88.

Yano, M. (2006), “A price competition game under free entry,” Economic Theory 29, 395-414. 